\title{
IAMJ
}

INTERNATIONAL

AYURVEDIC

MEDICAL JOURNAL

Review Article

ISSN: 2320-5091

Impact Factor: 6.719

\section{VITAMINS AND EYE: LITERATURE REVIEW}

Shardul Pathak ${ }^{1}$, Shambhavi Kulkarni²

${ }^{1}$ Asst. Professor, B.A.M.S, M.D (Dravyaguna), Siddhakala Ayurved Mahavidyalaya, Sangamner, Ahmadnagar, Maharashtra, India

${ }^{2}$ Naturopathy and Yoga Consultant, Clinical Nutritionist, B.N.Y.S., M.Sc Applied Nutrition and Dietetics

Sangamner, Ahmadnagar, Maharashtra, India

Corresponding Author: drshardulpathak@gmail.com

https://doi.org/10.46607/iamj2409102021

(Published Online: October 2021)

Open Access

(C) International Ayurvedic Medical Journal, India 2021

Article Received: 15/09//2021 - Peer Reviewed: 06/10/2021 - Accepted for Publication: 07/10/2021

\section{Check for updates}

\begin{abstract}
Diet has a long-term effect on eyes health. Several studies have proven that dietary intervention helps in age-related macular degeneration, glaucoma, and Diabetic retinopathy etc.

This review paper specifically focuses on the effects of Vitamins on the eye. Studies have shown Vitamins like A, $\mathrm{C}, \mathrm{D}$, as well as E has a key role to play in eye health. Ex. Vitamin A regulates the proliferation of corneal epithelial cells, and for enhancing the smoothness and stability of the ocular tear film. Vitamin D supplementation was shown to promote tear secretion. Vitamin C was significantly decreased in patients with Diabetic Retinopathy. Vitamin E in fighting against the progression of age-related macular diseases
\end{abstract}

Keywords: Eyecare, Vitamins, Diabetic Retinopathy, tear film.

\section{INTRODUCTION}

Diet has a long-term effect on eyes health. (Lawrenson 2019) ${ }^{1}$. Vitamins are known as micronutrients. Vitamin $\mathrm{A}$ is required to produce photoreceptor pigments in the retina, and it lack causes night vision problems. Vitamin A is also necessary for maintaining the health of the ocular surface. keratomalacia can result from a vitamin A deficiency in the diet. Vitamin A, like vitamins $\mathrm{C}$ and $\mathrm{E}$, has antioxidant properties. 
Vitamin $\mathrm{C}$ is secreted into the aqueous humour, which is higher in concentration than other body fluids, implying that it is especially important for the lens. Increased food intake raises the level of vitamin $\mathrm{C}$ in the aqueous, indicating that the lens will feel the effects of increased vitamin $\mathrm{C}$ consumption. Vitamins are thought to play a function in preserving the health of the retina and lens.

Both are at threat of oxidation damage caused by free radicals, which are produced in part by normal metabolic processes and in part by exposure to sunshine. Cataract formation has been linked to several systemic disorders, including cardiovascular, diabetic, renal, and gastrointestinal (diarrhoea) diseases, as well as increased mortalities. Similarly, hypertension is linked to age-related retinal macular degeneration (ARMD). It is possible that vitamins and other nutrients protect the lens and retina directly, or that they promote body health in a way that benefits the eye indirectly. The role of antioxidant vitamins in the prevention of vascular disease have been extensively researched.

\section{Effects of Vitamin A on eyes}

Vitamin A is made up of retinol, retinoic acid, and retinal and b carotene. Sources of Vitamin A are Cod liver, eggs, vegetables like leafy vegetables, sweet potato, carrot. Vitamin A deficiency can also lead to corneal dryness (xerosis), keratomalacia, and corneal ulceration, which are very common in children with poor diets. Tear film stability is very important for eyes to remain healthy and moist and to prevent dryness. The tear film consists of mucin, water, and lipid layers; however, it is precise structure, function, and biophysical and biochemical properties are complex.

Various studies have suggested an association between eye dryness and vitamin A deficiency. Vitamin $\mathrm{A}$ is an important component for the visual pigments and the integrity of the ocular surface, and it is used in the treatment of peripheral vision loss, age-related macular degeneration, retinitis pigmentosa, and superior limbic keratoconjunctivitis. Dry eye disease can result from vitamin A deficiency, with mild deficiency resulting in conjunctival changes and severe deficiency resulting in xerophthalmia. Vitamin A regulates the proliferation of corneal epithelial cells, and also enhances the smoothness and stability of the ocular tear film and supports tear production. (Alanazi et al., 2019) ${ }^{2}$

\section{Effects of Vitamin D on eyes}

Dry eye disease (DED), also known as dry eye syndrome (DES), keratoconjunctivitis sicca (KCS), and keratitis sicca, is a multifactorial disease of the tears and the ocular surface that results in discomfort, visual disturbance, and tear film instability with potential damage to the ocular surface. Dry eye disease is a common form of the ocular surface disease (OSD) and may overlap with other causes of OSD, such as ocular allergy and meibomian gland dysfunction. (Foster, 2019) ${ }^{3}$

Tear break-up time (TBUT), fluorescein staining score (FSS), and the Schirmer tear secretion test were used to evaluate the tear film. The study showed that vitamin D supplementation improved TBUT, FSS, eyelid margin hyperemia, and tear secretion. TBUT is the most frequently employed method for assessing tear instability. Tear film instability is linked to tear hyperosmolarity, which is considered a primary mechanism in the development of DES. Tear film instability and hyperosmolarity induce ocular surface damage and initiate an inflammatory cascade that generates innate and adaptive immune responses in DES. FSS and eyelid margin hyperemia are both associated with inflammation. FSS has been used to assess ocular surface damage using sodium fluorescein although it is not specific for DES. Eyelid margin hyperemia has been reported to contribute to DES and ocular surface disease. Tear secretion is a major factor in the development of DES. Reduced tear production results in tear instability and ocular surface damage.

Vitamin D supplementation was shown to promote tear secretion, reduce tear instability, and reduce inflammation of the ocular surface and eyelid margin. It has been suggested that DES is an autoimmune disease characterized by immune and inflammatory processes that affect the ocular surface. DES is an inflammatory disease that results from the activation of innate inflammatory pathways in resident ocular surface cells, as well as cytokines produced by recruited $\mathrm{T}$ helper 
(Th) cells. In this study, vitamin D supplementation reduced FSS and hyperemia of the eyelid margin. FSS is an indicator of ocular surface inflammation. Eyelid margin hyperemia is related to meibomian gland dysfunction and eyelid inflammation, which is a major cause of evaporative type DES. Vitamin D has extensive immunomodulatory effects. The production of TNF- $\alpha$ and IFN- $\gamma$ is significantly reduced by $1,25(\mathrm{OH}) 2 \mathrm{D} 3$ through interference with NF- $\kappa$ B production. 1,25(OH)2 $\mathrm{D} 3$ has immune regulatory effects on NK cell cytotoxicity, cytokine secretion, and the degranulation process as well as TLR4 expression38. Furthermore, 1,25(OH)2-Vitamin-D3 has been reported to attenuate Th17-related cytokines expression39. It has been reported that $1,25(\mathrm{OH}) 2 \mathrm{D} 3$ ameliorated the inflammation of the colon and spleen by downregulating the levels of Th 1 and Th17 cytokines. The Th17 concentration in tears has been reported to be significantly increased in DES patients, with the concentration associated with the disease severity. Another important cytokine in DES is IFN- $\gamma 1$. IFN- $\gamma$ is the signature cytokine from Th1 cells32. Increased IFN- $\gamma$ concentration in tears of DES patients has been previously reported41. Vitamin D can suppress inflammatory cytokines such as TNF, IL- 1, IFN- $\gamma$, and IL-2. (Bae et al., 2016) ${ }^{4}$

Vitamin D's ability to inhibit neovascularization also has led researchers to examine the hormone's involvement in diabetic retinopathy (DR) development. Serum vitamin $\mathrm{D}$ concentrations $\left(25 \mathrm{D}_{3}\right)$ were inversely related to the severity of retinopathy in diabetic patients, with the lowest concentrations of the hormone measured in patients with proliferative DR. Patients without associated retinopathy had the highest serum vitamin D concentrations. A similar study classified patients into diabetic groups based on disease severity and found that patients with proliferative DR had the lowest mean $25 \mathrm{D}_{3}$ levels $(21.1 \mathrm{ng} / \mathrm{mL})$. In addition, vitamin $\mathrm{D}$ deficiency was associated with an increased risk of retinopathy in an adolescent population with type 1 diabetes, however, it was not associated with changes in retinal geometric parameters such as vascular branching angle, length-diameter ratio, or tortuosity. (Cho, 2016)
A painless elevation of intraocular pressure (IOP) occurs, which can lead to progressive optic nerve damage and visual field loss. The mechanical theory of resistance to outflow (at the juxta canicular meshwork) is one postulated mechanism for glaucoma. Disturbances of trabecular meshwork (TM) collagen, TM endothelial cell dysfunction, basement membrane thickening, glycosaminoglycan deposits, narrowing intertrabecular spaces, and/or collapse of the Schlemm canal may occur. Experimental and clinical studies show that sustained elevation of IOP can cause optic nerve damage similar to primary open-angle glaucoma (POAG), thus providing support for the role of IOP. (Graham, 2020) ${ }^{6}$

Gene expression studies identified vitamin D as having the potential to modulate genes involved in regulating both aqueous humour outflow and production, as well as the architecture of the trabecular meshwork, thereby influencing IOP. In both cultured mouse calvarial cells and rat intestinal mucosa, treatment with vitamin D modulated the expression of numerous genes involved in intraocular pressure (IOP) regulation. Microarray analysis showed that $1,25 \mathrm{D}_{3} \mathrm{de}-$ creased the expression of carbonic anhydrase I (CAI), angiotensin I converting enzyme (ACE), aquaporin 1 channel (AQP1), and various cytoskeletal and extracellular matrix genes such as actin alpha (ACTA1) and fibronectin I. $1,25 \mathrm{D}_{3}$ also upregulated matrix metalloproteinases 3,11,13, and 14, as well as prostaglandin E receptor 4 for PGE2 (PTGER4), purinergic receptors P2Y and P2RY2, and chemokine (C-C motif) ligand 20 (CCL20). (Cho, 2016) ${ }^{5}$

\section{Role of Vitamin $\mathrm{E}$}

Cataracts are one of the commonest causes of significant vision loss in older people. They occur due to the accumulation of proteins damaged by free radicals. Several observational studies have revealed a potential relationship between vitamin E supplements and the risk of cataract formation. One study found that lens clarity was superior in participants who took vitamin E supplements and those with higher blood levels of the vitamin. In another study, long-term supplementation of vitamin E was associated with the slower progression of age-related lens opacification. However, in 
the randomised Age-Related Eye Disease Study (AREDS), vitamin E had no apparent effect on cataract development/progression over an average of 6.3 years. Overall, the available evidence is insufficient to conclude that vitamin E supplements, taken alone or in combination with other antioxidants, can reduce the risk of cataract formation(Rizvi et al., 2014) ${ }^{7}$

Vitamin $\mathrm{E}$ exists in four common forms in nature, namely, $\alpha$-tocopherol, $\beta$-tocopherol, $\delta$-tocopherol, and $\gamma$-tocopherol. It is an essential micronutrient and efficient antioxidant that scavenges free radicals. Deprivation of vitamin E could lead to lipofuscin accumulation, retinal damage, and loss of photoreceptors. Due to vitamin $\mathrm{E}$ being a fat-soluble vitamin, it plays an important role in fatty acid metabolism. Vitamin E is involved in the desaturation of PUFAs via the microsomal electron transport chain. In the human body, $\alpha$ tocopherol is the most abundant in both plasma and retinal tissue. Thus, studies suggested that increased dietary levels of vitamin $\mathrm{E}$ have been correlated with increased concentrations in the retina, and epidemiologic studies also suggest a beneficial effect of vitamin E for fighting the progression of age-related macular diseases.

\section{Role of Vitamin C}

Levels of vitamin $\mathrm{C}$ in the serum, aqueous humor, and vitreous humor were lower in patients with Diabetic retinopathy than those in non-diabetic patients. The mean concentration of ascorbate in the vitreous humor is $2 \mathrm{mM}$, whereas blood levels are only $50-60 \mu \mathrm{M}$, which is a 33- to 40-fold difference. The high concentration of vitamin $\mathrm{C}$ in the vitreous humor indicated that vitamin $\mathrm{C}$ acts as an antioxidant under high oxidative stress and has a protective effect on the retina, even in physiologically normal conditions. Vitamin C level in Proliferative diabetic retinopathy (PDR) measured only $11.1 \%$ of vitreous vitamin $\mathrm{C}$ levels, this indicates that increased oxidative stress in the eyes.

Retinal ischemia might be related to vitreous vitamin $\mathrm{C}$ depletion in patients with PDR. In this condition, vitreous vitamin $\mathrm{C}$ gets consumed faster to compensate for the increased oxidative stress that is produced on the ocular tissues. The vitreous humor metabolizes molecular oxygen in an ascorbate-dependent manner, thereby regulating intraocular oxygen tension. The vitreous humor is also important in the development of cataracts and primary open-angle glaucoma. In this study, Diabetic macular ischaemia (DMI) as an ischemic marker, was associated with vitreous vitamin $\mathrm{C}$ depletion in PDR patients. In addition, correlations between vitamin $\mathrm{C}$ levels in the serum, aqueous humor, and vitreous humor were not significant in patients with PDR. However, vitreous vitamin C was significantly decreased in patients with PDR. These results implied that ocular factors, especially retinal ischemia, are more prevalent than any systemic factors in decreasing vitreous vitamin C in PDR patients. (Park et al., 2019) ${ }^{8}$

\section{DISCUSSION}

Vitamin A deficiency can lead to corneal dryness (xerosis), keratomalacia, and corneal ulceration. Studies have suggested a link between eye dryness and vitamin A deficiency. Vitamin A used in the treatment of peripheral vision loss, age-related macular degeneration, retinitis pigmentosa, and superior limbic keratoconjunctivitis. It regulates the proliferation of corneal epithelial cells and enhances the smoothness and stability of the ocular tear film and supports tear production.

Vitamin D plays a key role in Dry Eye Syndrome (DES). Vitamin D supplementation was shown to promote tear secretion, reduce tear instability, and reduce inflammation of the ocular surface and eyelid margin. The study showed that vitamin D supplementation improved Tear break-up time (TBUT), fluorescein staining score (FSS), and the Schirmer tear secretion test, eyelid margin hyperemia, and tear secretion.

Vitamin D's ability to inhibit neovascularization also has led researchers to examine the hormone's involvement in diabetic retinopathy (DR) development.

Deprivation of vitamin E could lead to lipofuscin accumulation, retinal damage, and loss of photoreceptors. Studies suggested that increased dietary levels of vitamin $E$ have been correlated with increased concentrations in the retina and fighting the progression of age-related macular diseases. About Vitamin E and cataracts, the available evidence is insufficient to con- 
clude vitamin E supplements, taken alone or in combination with other antioxidants, can reduce the risk of cataract formation(Rizvi et al., 2014) ${ }^{7}$

The high concentration of vitamin $\mathrm{C}$ in the vitreous humor indicated that vitamin $\mathrm{C}$ acts as an antioxidant under high oxidative stress and has a protective effect on the retina. ocular factors, especially retinal ischemia, are more prevalent than any systemic factors in decreasing vitreous vitamin $\mathrm{C}$ in PDR patients. (Park et al., 2019) ${ }^{8}$

\section{CONCLUSION}

Diet has a long-term effect on eyes health. Several studies have proven that dietary intervention helps in age-related macular degeneration, glaucoma, and Diabetic retinopathy etc.

Vitamin A regulates the proliferation of corneal epithelial cells and enhances the smoothness and stability of the ocular tear film and supports tear production. Vitamin C was significantly decreased in patients with Diabetic Retinopathy. Vitamin D supplementation was shown to promote tear secretion, reduce tear instability, and reduce inflammation of the ocular surface and eyelid margin. Studies also suggest a beneficial effect of vitamin $\mathrm{E}$ for fighting the progression of age-related macular diseases.

\section{REFERENCES}

1. Lawrenson, J. G., \& Downie, L. E. (2019). Nutrition and Eye health. Nutrients, 11(9), 11-14. https://doi.org/10.3390/nu11092123

2. Alanazi, S. A., El-Hiti, G. A., Al-Baloud, A. A., Alfarhan, M. I., Al-Shahrani, A., Albakri, A. A., Alqahtani, S., \& Masmali, A. M. (2019). Effects of short-term oral vitamin A supplementation on the ocular tear film in patients with dry eye. Clinical Ophthalmology, 13, 599-604. https://doi.org/10.2147/OPTH.S198349

3. C Stephen Foster(2019) Dry Eye Disease(Keratoconjunctivitis Sicca) https://emedicine.medscape.com/article/1210417overview\#a2

4. Bae, S. H., Shin, Y. J., Kim, H. K., Hyon, J. Y., Wee, W. R., \& Park, S. G. (2016). Vitamin D Supplementation for Patients with Dry Eye Syndrome Refractory to Conventional Treatment. Scientific
Reports, 6(July), 1-10. https://doi.org/10.1038/srep33083

5. Cho. (2016). HHS Public Access. Physiology \& Behavior, 176(1), 100-106. https://doi.org/10.1016/j.exer.2015.02.019.

6. Graham, Robert H (2020) emedicine.medscape Glaucoma, Suspect, Adult https://emedicine.medscape.com/article/1205421overview\#a5

7. Rizvi, S., Raza, S. T., Ahmed, F., Ahmad, A., Abbas, S., \& Mahdi, F. (2014). The role of Vitamin E in human health and some diseases. Sultan Qaboos University Medical Journal, 14(2), 157-165.

8. Park, S. W., Ghim, W., Oh, S., Kim, Y., Park, U. C., Kang, J., \& Yu, H. G. (2019). Association of vitreous Vitamin $\mathrm{C}$ depletion with diabetic macular ischemia in proliferative diabetic retinopathy. PLoS ONE, 14(6), 1-11. https://doi.org/10.1371/journal.pone.0218433

\section{Source of Support: Nil \\ Conflict of Interest: None Declared}

How to cite this URL: Shardul Pathak \& Shambhavi Kulkarni: Vitamins And Eye: Literature Review. International Ayurvedic Medical Journal \{online\} 2021 \{cited October 2021\} Available from:

http://www.iamj.in/posts/images/upload/2445_2449.pdf 